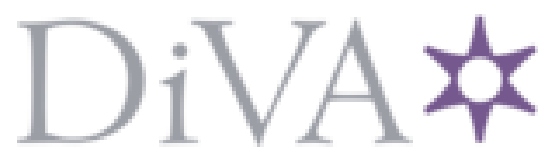

http://www.diva-portal.org

\title{
Preprint
}

This is the submitted version of a paper published in Evolution \& Development.

Citation for the original published paper (version of record):

Janssen, R., Jörgensen, M., Prpic, N., Budd, G. (2014)

Aspects of dorso-ventral and proximo-distal limb patterning in onychophorans.

Evolution \& Development, 17(1): 21-33

http://dx.doi.org/10.1111/ede.12107

Access to the published version may require subscription.

N.B. When citing this work, cite the original published paper.

Permanent link to this version:

http://urn.kb.se/resolve?urn=urn:nbn:se:uu:diva-246052 


\section{Aspects of dorso-ventral and proximo-distal limb patterning in onychophorans}

Ralf Janssen $^{* 1}$, Mette Jörgensen ${ }^{1}$, Nikola-Michael Prpic ${ }^{2}$, and Graham E. Budd ${ }^{1}$

${ }^{1}$ Uppsala University, Department of Earth Sciences, Palaeobiology, Villavägen 16, 75236 Uppsala, Sweden

2 Abteilung für Entwicklungsbiologie, Johann-Friedrich-Blumenbach-Institut für Zoologie und Anthropologie, Georg-August-Universität Göttingen, GZMB, ErnstCaspari-Haus, Justus-von-Liebig-Weg 11, 37077, Göttingen, Germany

* Author for correspondence:

Ralf Janssen

Phone: $\quad(++46) 184712763$

e-mail: $\quad$ ralf.janssen@geo.uu.se

Word count: $\quad 7304$

Figures: $\quad 7$

Tables:

Supplementary data: 2 additional figures + figure legends

Key Words: Onychophora, Arthropoda, limb development, evolution, lobopod 


\begin{abstract}
Onychophorans (velvet worms) are the sister group of the arthropods, but their limb morphology represents a stage before arthropodization (i.e. the segmentation of the limbs). We investigated the expression of onychophoran homologs of genes that are involved in dorso-ventral (DV) and proximo-distal (PD) limb patterning in arthropods. We find that the two onychophoran optomotor-blind (omb) genes, omb-1 and $o m b-2$, are both expressed in conserved patterns in the dorsal ectoderm of the limbs, including the onychophoran antennae (the frontal appendages). Surprisingly, the expression of decapentaplegic ( $d p p)$, that acts upstream of omb in arthropods, is partially reversed in onychophoran limbs compared to its expression in arthropods. A conserved feature of $d p p$ expression in arthropods and onychophorans, however, is the prominent expression of $d p p$ in the tips of developing limbs, which therefore may represent the ancestral pattern. The expression patterns of wingless $(w g)$ and $H 15$ are very diverged in onychophorans. The $w g$ gene is only expressed in the limb tips and the single $H 15$ gene is expressed in a few dorsal limb cells, but never on the ventral side. The expression of $w g$ and $d p p$ at the limb tips is one of the three possible alternatives predicted by the topology model of arthropod limb patterning and is thus compatible with a conserved function of $w g$ and $d p p$ in the patterning of the PD axis. On the other hand DV limb patterning is less conserved, and the specification of ventral fate appears to involve neither $w g$ nor $H 15$ expression.
\end{abstract}




\section{INTRODUCTION}

The phylum Onychophora comprises less than 200 described species (Oliveira Ide et al. 2012). The arthropods, the most closely related phylum, however, represent one of the most speciose animal phyla. Estimations suggest that there are more than ten million species belonging to four (or five, depending on the status of the pycnogonids) arthropod classes, Chelicerata (with or without Pycnogonida), Myriapoda, Crustacea and Insecta. A unique hallmark, and indeed the eponymous feature of the arthropods, is the presence of segmented (jointed) appendages. In contrast, onychophorans possess appendages that are not jointed. Such limbs are often referred to as "oncopodia" or "lobopodia". The evolution of jointed limbs is thought to represent one of the key events in the arthropod stem that subsequently led to the massive radiation of the arthropods.

The development of arthropod limbs is organized along the proximal-distal (PD), the anterior-posterior (AP), and the dorso-ventral (DV) axis. In the fly Drosophila melanogaster the AP axis is determined during segment patterning via the function of segment-polarity genes (Morata and Lawrence 1975; Cohen 1990; Brook 2010). This function is most probably conserved in all arthropods studied so far as indicated by gene expression studies (e.g. Abzhanov and Kaufman 2000; Damen 2002; Prpic et al. 2003). The PD axis is established via the activation of the limb gap-genes Distal-less $(D l l)$ and dachshund (dac), and the repression of the limb gap-genes homothorax (hth) and extradenticle (exd), both of which is orchestrated by the morphogens Wingless (WG) and Decapentaplegic (DPP) (Campbell et al. 1993; Diaz-Benjumea et al. 1994; Jiang and Struhl 1996; Lecuit and Cohen 1997; Abu-Shaar and Mann 1998; Estella and Mann 2008). Again, gene expression data suggest that the function of limb gap genes is widely conserved among arthropods (e.g. Abzhanov and Kaufman 2000; 
Inoue et al. 2002; Prpic et al. 2001, 2003; Prpic and Tautz 2003; Angelini and Kaufman 2005; Tanaka and Truman 2007; Pechmann and Prpic 2009; Sharma et al. 2012; Schaeper et al. 2013), and even in an onychophoran (Janssen et al. 2010). In Drosophila, the same morphogenes, DPP and WG, are involved in the formation of the DV axis (Struhl and Basler 1993; Brook and Cohen 1996, Morimura et al. 1996). The $w g$ gene is expressed along the ventral side of the leg imaginal discs and loss of WG leads to the loss of ventral features and dorsalization of the limbs (Struhl and Basler 1993; Couso et al. 1993; Wilder and Perrimon 1995; Zecca et al. 1996). Thus, WG is required for ventral fate specification and it controls this process by the activation of the two T-box genes, H15 and midline (mid)) in ventral cells of the disc (Brook and Cohen 1996; Svendsen et al. 2009). H15 and mid are paralogous copies and show very similar nucleotide sequences and expression patterns, but only mid acts as selector gene in ventral tissue, because only mid can induce ventral fate if it is experimentally misexpressed on the dorsal side of the disc (Svendsen et al. 2009). Similar to $w g$ on the ventral side, $d p p$ is expressed along the dorsal side of the leg imaginal disc and loss of DPP leads to the loss of dorsal features and ventralization of the limbs (Held et al. 1994; Maves and Schubiger 1998). Similar to WG that activates H15 and mid in the ventral ectoderm, DPP activates the expression of another T-box gene, optomotor-blind (omb), in the dorsal ectoderm (Nellen et al. 1996; Brook and Cohen 1996; Grimm and Pflugfelder 1996; Maves and Schubiger 1998). Analogous to $\mathrm{mid}, \mathrm{omb}$ can induce dorsal fate if misexpressed in ventral cells of the leg disc, and $o m b$ is even capable of activating $d p p$ expression in these cells (Maves and Schubiger 1998). Thus, although omb is initially controlled by DPP, it is also capable of activating the entire dorsalization program on its own. Comparative gene expression data and, for $w g$, functional data suggest that the function of ventral and dorsal 
patterning genes, in particular the role of $H 15 / \mathrm{mid}$ and $\mathrm{omb}$, is conserved in all arthropods (e.g. Prpic et al. 2003, 2005; Janssen et al. 2008a; Grossmann et al. 2009, but see Ober and Jockusch 2006 and Angelini and Kaufman 2005 for a different opinion).

We therefore wished to address the question whether this genetic network is possibly conserved in the simple appendages of onychophorans as well, or whether it evolved in the lineage leading to the arthropods.

In the present study we investigated the expression profiles of $d p p, w g$, two omb paralogs and one single $H 15$ paralog in the onychophoran Euperipatoides kanangrensis (and for $\mathrm{H} 15$ also in a second onychophoran species, Cephalofovea clandestina). Our data reveal conserved expression of omb genes, but also diverged expression of $H 15, w g$ and $d p p$, suggesting that at least the mechanisms of ventral limb fate specification are specific to the jointed limbs of the arthropods. Because this arthropod ventral mechanism is at the same time involved in many aspects of PD axis patterning (including the orchestration of limb segmentation), the advent of the arthropod-mode of ventral fate specification after the split between arthropods and onychophorans may also represent one prerequisite for the subsequent evolution of the arthropods jointed limbs.

\section{MATERIAL AND METHODS}

Animal husbandry and embryo preparation

Females of Euperipatoides and Cephalofovea were collected in Kanangra Boyd National Park, NSW, Australia. Embryos were dissected from the females from September to December to obtain a series of developmental stages to be used later for 
the in-situ hybridization experiments (Janssen and Budd 2013). Each female carries approximately 50 (sometimes more) embryos of different consecutive developmental stages. The embryonic chorion and the vitelline membrane were removed manually with Dumont size 5 forceps and the embryos were fixed in $4 \%$ formaldehyde in PBST (0.1M phosphate buffered saline with $0.1 \%$ Tween- 20 at $\mathrm{pH} 7.4)$ for approximately four hours at room temperature. The embryos were then dehydrated in $100 \%$ methanol and stored at $-20^{\circ} \mathrm{C}$ for at least one month before using them in hybridization experiments.

\section{RT-PCR and gene cloning}

RNA isolation and cDNA synthesis were performed as described in Janssen et al. (2004). Gene fragments of Euperipatoides optomotor-blind1 (omb1), optomotorblind2 (omb2), H15 and decapentaplegic ( $(\mathrm{pp}$ ) have been isolated by PCR using the degenerate primers described in Prpic et al. (2003). Longer fragments of these genes were then obtained by RACE (GeneRacer cDNA amplification Kit, Invitrogen). The fragment of Cephalofovea H15 was isolated with the primers fw1 (GGNACNGARATGATHATHAC) and bw1 (TTNARYTTNGTNATNARYTCRTT) in a first PCR, and the primers fw2 (CCNGTNGAYAAYAATMGNTA) and bw2 (GCNGTRAANACNGTYTCNGG) in a nested PCR. All fragments were cloned into the PCRII vector (Invitrogen). Sequences of the fragments were determined by means of Big Dye chemistry on an ABI3730XL sequence analyser by a commercial sequencing service (Macrogen). The sequences are available from the EMBL

nucleotide database under Accession Numbers HG326421 (Ek-omb1), HG326422 (Ek-omb2), HG326425 (Ek-dpp), HG326423 (Ek-H15), and HG326424 (Cc-H15). 
In-situ hybridization, cell nuclei staining, staging, and data documentation

In-situ hybridization experiments were performed as described previously (Janssen et al. 2010). Detection of the $d p p$-signal required extended staining time for 12 to 36 hours at room temperature; usually staining with most other probes only takes one to four hours. During prolonged staining durations the staining-solution was exchanged every six hours. A set of $d p p$-probes representing different parts of the gene was used to determine the probe giving the best results (Fig. S1). Digoxigenin (DIG) or Fluorescein (FL) labelled RNA probes were transcribed from the cloned gene fragments. Cell nuclei were stained with $1 \mu \mathrm{g} / \mathrm{ml}$ DAPI (4-6-Diamidino-2phenylindole) in PBST for 20 minutes. Excessive DAPI was removed by several washes in PBST. Embryonic stages from the formation of the germ disc to formation of the cuticle are described in the supplementary data of Janssen and Budd (2013). Embryos were analysed under a Leica dissection microscope equipped with a Leica DC100 digital camera. Brightness, contrast and colour values were adjusted in all images using the image processing software Adobe Photoshop CS2 (Version 9.0.1 for Apple Macintosh).

\section{Phylogenetic analysis}

Sequences were compared to orthologs via BLAST search, and aligned in Clustal_X (Thompson et al. 1997) (BLOSUM matrix (Henikoff and Henikoff 1992)). Maximum likelihood analysis was performed with the Quartet Puzzling method (Strimmer and von Haeseler 1996) as implemented in PAUP 4.0 b10 (Swofford 2002) (Fig. S2).

\section{RESULTS}


The onychophoran T-box genes optomotor-blind1 (omb1), optomotor-blind2 (omb2) and H15, and the TGF-beta family gene decapentaplegic (dpp)

We isolated two optomotor-blind type genes from Euperipatoides by means of RTPCR with degenerate primers. These two genes also represent the only omb-type genes found in an embryonic transcriptome (assembled from Illumina HiSeq2000 reads) that became available after the RT-PCR analysis had been performed (Janssen and Budd 2013), suggesting that only these two genes are transcribed during embryonic development. Both genes, which we designate as $o m b 1$ and $o m b 2$, cluster with omb-orthologs from Drosophila and other arthropods in a well-supported group, and to the exclusion of other related T-box genes (Fig. S2). The omb1 gene branches at the base of arthropod $o m b$-genes, while $o m b 2$ branches within the arthropod genes. Our analysis, including several independent RT-PCR screens for H15-type genes in the onychophorans Euperipatoides and Cephalofovea, and the sequenced embryonic transcriptome of Euperipatoides, reveal the presence of only one single H15-type gene in onychophorans. This is notable, because two H15-type genes exist in arthropods including chelicerates, a myriapod and insects, (summarized in Janssen et al. 2008a). Consistent with the phylogenetic position of onychophorans as sister group to the arthropods, the single onychophoran H15 gene branches at the base of the arthropod $H 15$ genes.

The single Euperipatoides $d p p$ gene clusters with $d p p$ genes from other arthropods and the mouse Bone morphogenic factor 2 (Bmp2), and clearly to the exclusion of other BMP-like genes from Euperipatoides, glass bottom boat (gbb) and antidorsalizing morphogenetic protein-like (admp). 
Euperipatoides $d p p$ is expressed in most cells of the embryonic body at a low level (Fig. 1A). However, in the developing limbs, including the frontal appendages, $d p p$ is expressed stronger, except for ectodermal cells along the anterior side (Fig. 1C-E) and along the dorsal side (Fig. 6A-H). This is especially clear in late-stage embryos (Fig. 6E-H). Very strong expression is found in the tips of all appendages, except for the frontal appendages (Figs 1B-E and 6A-H). At late embryonic stages, $d p p$ is also expressed in a differential pattern in the developing ventral nervous system, the head lobes and in cells along the lateral rim of the germ band, which will fuse over the yolk during dorsal closure and contribute to the heart (Fig. 1A, B).

We note that this expression profile of $d p p$ in Euperipatoides kanangrensis deviates in some aspects from the pattern of $d p p$ described for the closely related species Euperipatoides rowelli (Treffkorn and Mayer 2013). This might point to $d p p$ patterning differences between very closely related onychophoran species. Alternatively, the discrepancy might be caused by different efficiencies of wholemount in situ hybridisation methodology. Indeed, we note that the $d p p$ signal in $E$. rowelli is generally much weaker than the $d p p$ signal that we obtained in $E$. kanangrensis. Such differences in the sensitivity of $d p p$ mRNA detection might explain the presence of some expression domains in E. kanangrensis, that appear to be absent in E. rowelli, e.g. the distal domain in all appendages.

\section{Expression of the wingless gene of Euperipatoides kanangrensis}

Expression of Euperipatoides wg has previously been described in detail in Eriksson et al. (2009). Note that $w g$ is expressed in continuous stripes throughout the ventral side of the limbs early during limb developmental (Fig. 2). 
Expression of Euperipatoides kanangrensis optomotor-blind1 and optomotor-blind2

Early expression of $\mathrm{ombl}$ is restricted to a ring around the posterior pit region (Fig. 3A). Subsequently, expression appears in the posterior and dorsal portions of the head lobes (Fig. 3A). At later stages ombl is strongly expressed in dorsal tissue of the frontal appendages, the ocular region in the dorsal part of the head lobes, and along the dorsal side of all other appendages (Figs 3 and 6I-L). Expression in the limbs, including the frontal appendages, remains throughout development (Fig. 3H, I). At late embryonic stages $o m b 1$ is expressed in an arch-shaped domain in the head lobes that probably corresponds to the developing optical centers of the brain (Fig. 3H, I). omb2 appears first in the posterior and dorsal region of the head lobes (Fig. 4A); it is not expressed in a ring around the posterior pit. Soon after, omb2 is expressed in form of a continuous anterior-posterior stripe along the dorsalmost rim of the developing germ band (Fig. 4B). When the limb buds start to grow out, it becomes clear that this expression is in the dorsal part of the limbs and in the dorsal tissue between the limbs (Figs 4C and 6M-P); these stripes from the left and right halves of the germ band converge in the posterior pit region (Fig. 3D). The domain of omb2 in the head lobes is larger than that of $o m b 1$ and reaches further ventrally (cf. Figs 3D, E and 4C-F). At approximately stage 18, expression appears in form of a dot dorsal to the center of the limbs in all trunk segments (Fig. 4F). In subsequent stages omb2 is expressed in the developing ventral nervous system (Fig. 4G) and in a complex pattern in the outgrowing dorsal tissue (Fig. 4I). Notably, at later stages omb2 expression is weaker in the distal part of the limbs (Fig. 4H). This is different from the expression of $o m b l$.

Expression of Euperipatoides kanangrensis H15 and Cephalofovea clandestina H15

The expression of Euperipatoides H15 and Cephalofovea H15 (not shown) is 
identical. At early developmental stages H15 is expressed in the posterior part of the mesoderm of all limbs, except the frontal appendages (Fig. 5A, B). At slightly later stages it becomes clear that H15 is expressed stronger in L4 and L5 (Fig. 5C, D). This increased expression may be related to the development of the anlagen of the nephridia in these segments, because the nephridia of L4 and L5 are significantly larger than the others (Mayer 2006). However, also the jaws express H15 in a similar way as the other trunk segments, although there is no rudiment of the nephridial anlagen in the jaw-bearing segment (Figs. 5A, C, D and 6Q-T) (Mayer 2006). At later stages, $H 15$ is expressed in the dorsal mesoderm at the base of all limbs (except the frontal appendages), and in a smaller ectodermal patch dorsally at the very base of the limbs (Fig. 5R-T). Apart from the expression in the limbs, $H 15$ is also expressed in the developing heart and in the ventral nervous system at later stages (Fig. 5C, D). Expression of $H 15$ genes in the heart and the ventral nervous system is conserved in onychophorans and arthropods (Griffin et al. 2000; Janssen and Damen 2008; Miskolczi-McCallum et al. 2005; Prpic et al. 2005).

\section{DISCUSSION}

Dorsal limb patterning in arthropods and onychophorans

In Drosophila, the morphogens DPP and WG are crucial for the formation of the dorsoventral limb-axis (Struhl and Basler 1993; Brook and Cohen 1996; Morimura et al. 1996; summarized in Brook 2010). The DPP protein is produced in a thin sector of dorsal cells in the leg imaginal disc and also moves from this area of synthesis to neighboring cells up to a few cell diameters away. In this area of its influence, DPP activates the expression of the T-box gene optomotor-blind $(\mathrm{omb})$, which is therefore 
expressed in a broader dorsal sector of the leg imaginal disc (Grimm and Pflugfelder 1996; Maves and Schubiger 1998). This dorsal expression domain suggests that omb is involved in the specification of dorsal fate. Indeed, misexpression of omb elsewhere in the disc leads to a transdetermination of these areas into dorsal fate (Maves and Schubiger 1998). The expression of omb along the dorsal side of the limbs is highly conserved among arthropods (Prpic et al. 2003, 2005; Janssen et al. 2008a), and our present results show that also in onychophorans both paralogous copies of omb are expressed along the dorsal side of all limb types. However, despite its conserved expression pattern, the mode of its activation by DPP is unlikely to be evolutionarily conserved. Data from a growing number of arthropods shows that only in a few species $d p p$ is actually expressed in a dorsal stripe or sector like in Drosophila. In fact, Drosophila melanogaster is the only species for which the interplay between $d p p$ and $o m b$ in dorsal fate specification has been demonstrated. In the cricket Gryllus bimaculatus, the grasshopper Schistocerca americana, and the pill millipede Glomeris marginata, $d p p$ is only transiently expressed along the dorsal side of the limbs, and later resolves into a distal spot of expression and/or segmental rings (Niwa et al. 2000; Jockusch et al. 2000; Prpic 2004). In other arthropods including the spider Cupiennius salei (Prpic et al. 2003), the beetle Tribolium castaneum (Sanchez-Salazar et al. 1996; Ober and Jockusch 2006), the sawfly (despite its name a hymenopteran) Athalia rosae (Yamamoto et al. 2004) and the true bug Oncopeltus fasciatus (Angelini and Kaufman 2005) $d p p$ only shows the expression in the tips and the segmental rings, but a dorsal stripe or sector is lacking even at early stages of limb development. These findings are compatible with our findings in Euperipatoides, that also lacks dorsal $d p p$ expression, but has a distal expression domain in all limb types, except for the frontal appendage. Taken together, these data suggest that dorsal fate specification via the 
expression of $o m b$ in the limbs was already present before the separation of arthropods and onychophorans. However, its control via DPP appears to be a later addition to the dorsal specification mechanism, that has evolved for the imaginal discs of Drosophila, and possibly independently also in a few arthropods that show transient dorsal $d p p$ expression in the limbs.

\section{Ventral limb patterning in arthropods and onychophorans}

In Drosophila leg imaginal discs, the morphogen WG and the T-box transcription factors $H 15$ and mid interact on the ventral side in a similar way as DPP and $o m b$ do on the dorsal side. WG protein is produced in a thin ventral sector of the leg imaginal disc and moves from there to neighboring ventral cells. In these cells, WG activates the transcription of H15 and mid (Brook and Cohen 1996; Svendsen et al. 2009), which are therefore expressed in a broad ventral sector of the disc. Gene function studies show that $w g$ is indeed essential for ventral fate determination in the Drosophila leg imaginal disc (Struhl and Basler 1993; Couso et al. 1993; Wilder and Perrimon 1995; Zecca et al. 1996; Brook and Cohen 1996). Similar to omb in dorsal fate specification, mid (but not H15) can induce ventral fate if expressed elsewhere in the leg disc (Svendsen et al. 2009). H15 appears to be involved in ventral cell patterning, but is not sufficient for ventral fate specification. Consistent with an essential role for $w g$ in ventral limb specification, comparative studies show that the ventral stripe or sector in the developing limbs is one of the most conserved expression patterns in all arthropods (e.g. Nulsen and Nagy 1999; Miyawaki et al. 2004; Janssen et al. 2004; Janssen et al. 2008b) and recent functional studies also support a role of $w g$ in ventral limb development in at least (holometabolous) insects (Ober and Jockusch 2006; Grossmann et al. 2009, but see Angelini and Kaufman 
2005 for contradicting results on a hemimetabolous insect). Surprisingly, wg expression in Euperipatoides deviates from the typical arthropod expression pattern. A continuous ventral stripe from the tip to the base is only present in early leg buds. Soon after, this continuous stripe splits into a spot in the leg tips and a ventral stripe in the body segment. The cells along the ventral side of the developing leg (and all other appendage types) do not express $w g$ during the further growth of the limbs. This could indicate that $w g$ is not required to specify the fate of these ventral cells in onychophorans. Alternatively, the short-lived ventral expression in the early limb bud could be sufficient to specify ventral fate, and this information is later maintained in the offspring of all ventral cells by other factors. Indeed, studies in the legs of the beetle Tribolium suggest that the roles of $w g$ in specifying ventral fate, and in orchestrating proximodistal development are not only mechanistically, but also temporally separate (Grossmann et al. 2009).

The role of H15-type genes in ventral fate specification in Drosophila leg imaginal discs is more variable, because only one of the two paralogous copies of this gene (i.e. mid) is sufficient for ventral fate specification (Svendsen et al. 2009). This is compatible with gene expression studies in a variety of arthropod species including insects, myriapods and chelicerates (e.g. Janssen et al. 2004, 2008a; Prpic et al. 2005), that show that the expression pattern of $\mathrm{H} 15$ is less conserved among the arthropods than the highly conserved $w g$ expression pattern. In addition, in most species the $H 15$ gene is also present in two or more paralogous copies that may show very different expression patterns (e.g. Janssen et al. 2008a). Nevertheless, all of the known arthropod H15 paralogs show expression in at least some ventral limb cells, and at least one of the paralogs is expressed along most of the ventral surface of the legs. 
This indicates that the ancestral $H 15$ gene indeed had a role in ventral patterning. However, the expression of H15 in Euperipatoides does not support an ancestral role of $H 15$ in ventral limb specification. The single $H 15$ homolog of Euperipatoides is not expressed in ventral cells in any limb type. By contrast, it is expressed in a proximal subset of dorsal cells, and this rather indicates a role in dorsal limb development. Thus, if the partial ventral expression found in all arthropods really traces from an ancestral function in ventral limb fate specification, then this role must have evolved in the arthropod ancestor after the split between arthropods and onychophorans.

The ancestral role of Dpp/Wg signaling in proximodistal limb axis specification At first glance, the limbs of onychophorans are simple tube-shaped outgrowths with only little differentiation along the proximodistal axis. However, although the limbs of onychophorans are not jointed, their epidermis shows repeated thickenings (annulation), and the limbs possess accurately spaced ring-nerves and are equipped with sensory structures along the PD axis (e.g. Walker and Tait 2004; Mayer and Whitington 2009). These features demand regionalization of the limbs, likely by the so-called limb gap gene orthologs Distal-less (Dll), dachshund (dac), homothorax (hth) and extradenticle (exd) (Janssen et al. 2010). It is unclear, however, whether the upstream regulation of these genes is similar in onychophorans and arthropods. In the Drosophila leg imaginal disc, the WG and DPP proteins produce two partially overlapping protein gradients (Lecuit and Cohen 1997; reviewed in Estella et al. 2012). Each cell in the disc receives a different amount of these two proteins and thus can use the concentration of both proteins as a kind of coordinate system or unique "ZIP code" to establish its location in the disc epithelium and to activate the 
appropriate target genes along the proximodistal limb axis (e.g. Lecuit and Cohen 1997). This system of proximodistal axis patterning is obviously adapted to the flat topology of the leg imaginal disc in Drosophila and might therefore not be present in the tube-shaped limb buds of other arthropods. Indeed, the expression pattern of $d p p$ is in most arthropods not similar to the Drosophila condition. However, a model has been proposed ("topology model") that argues for a conserved mode of DPP and WG action, and suggests that the differences in $d p p$ expression do not indicate any mechanistic differences, but only account for the differences in topology between a flat leg disc and a tube-shaped limb bud (Prpic et al. 2003). According to the topology model, if the Drosophila leg disc expression patterns of $w g$ and $d p p$ were directly transferred to a tube-shaped limb bud, then the resulting protein gradients would not combine correctly and therefore lead to a mispatterning along the proximodistal axis. In a tube-shaped limb bud, the gradients of WG and DPP only combine in a way that leads to a correct patterning of the proximodistal axis if either $w g$ or $d p p$ expression, or the expression of both genes, is restricted to the distal limb tip. Indeed, a strong expression of $d p p$ restricted to the limb tip is observed in all arthropods with tubeshaped limb buds. The alternative, the restriction of $w g$ expression to the limb tip, has not yet been observed in an arthropod species, probably because $w g$ has an additional role during ventral limb development and therefore cannot be removed from ventral cells (see also discussion above). Intriguingly, in Euperipatoides the expression of both genes, $w g$ and $d p p$, is restricted to the tip of the limbs, representing the third alternative predicted by the topology model. This opens the possibility that in onychophorans, the morphogens WG and DPP organize proximodistal patterning via fully overlapping gradients emanating from the limb tip, and that these distal cells therefore comprise a localized organizer for proximodistal axis formation in 
onychophoran limbs.

Mapping the expression patterns on the phylogenetic tree of the arthropods and onychophorans (Fig. 7) suggests that the restriction of $d p p$ and $w g$ expression to the distal limb tip is the ancestral condition. Expansion of $w g$ expression along the ventral side coincides with the split between onychophorans and arthropods (and is probably connected with the origin of a ventral role of $w g$ in arthropod limbs), whereas the expansion of $d p p$ expression along the dorsal side appears to be connected with the evolution of flat imaginal discs in dipterans (the functional significance of the transient dorsal expression in the only myriapod species studied so far is unclear).

\section{ACKNOWLEDGEMENTS}

This work has been supported by the Swedish Research Council (VR: grant to GEB), the European Union via the Marie Curie Training network "ZOONET" (MRTN-CT2004-005624 (to GEB and RJ)). The work of NMP is supported by the German Research Council (DFG; grant number PR 1109/6-1). We gratefully acknowledge the support of the NSW Government Department of Environment and Climate Change by provision of a permit SL100159 to collect onychophorans at Kanangra Boyd National Park, and to the Australian Government Department of the Environment, Water, Heritage and the Arts for export permits WT2009-4598 and WT2012-4704. Jean Joss, Rolf Ericsson, Robyn Stutchbury, and especially Noel Tait, were of great help during the onychophoran collection trip.

\section{REFERENCES}


Abu-Shaar, M., Mann, R. S. 1998. Generation of multiple antagonistic domains along the proximodistal axis during Drosophila leg development. Development 125: $3821-3830$.

Abzhanov, A., Kaufman, T. C. 2000. Homologs of Drosophila appendage genes in the patterning of arthropod limbs. Dev. Biol. 227: 683-689.

Angelini, D. R., Kaufman, T. C. 2005. Functional analyses in the milkweed bug Oncopeltus fasciatus (Hemiptera) support a role for Wnt signaling in body segmentation but not appendage development. Dev. Biol. 283: 409-423.

Brook, W. J., Cohen, S. M. 1996. Antagonistic interactions between Wingless and Decapentaplegic responsible for dorsal-ventral pattern in the Drosophila leg. Science 273: 1373-1377.

Brook, W. J. 2010. T-box genes organize the dorsal ventral leg axis in Drosophila melanogaster. Fly (Austin) 4: 159-162.

Campbell, G., Weaver, T., Tomlinson, A. 1993. Axis specification in the developing Drosophila appendage: the role of wingless, decapentaplegic, and the homeobox gene aristaless. Cell 74: 1113-1123.

Cohen, S. M. 1990. Specification of limb development in the Drosophila embryo by positional cues from segmentation genes. Nature 343: 173-177.

Couso, J. P., Bate, M., Martinez-Arias, A. 1993. A wingless-dependent polar coordinate system in Drosophila imaginal discs. Science 259: 484-489.

Damen, W. G. M. 2002. Parasegmental organization of the spider embryo implies that the parasegment is an evolutionary conserved entity in arthropod embryogenesis. Development 129: 1239-1250.

Diaz-Benjumea, F. J., Cohen, B., Cohen, S. M. 1994. Cell interaction between compartments establishes the proximal-distal axis of Drosophila legs. Nature 
Eriksson, B. J., Tait, N. N., Budd, G. E., Akam, M. 2009. The involvement of engrailed and wingless during segmentation in the onychophoran Euperipatoides kanangrensis (Peripatopsidae: Onychophora) (Reid 1996). Dev. Genes Evol. 219: 249-264.

Estella, C., Mann, R. S. 2008. Logic of Wg and Dpp induction of distal and medial fates in the Drosophila leg. Development 135: 627-636.

Estella, C., Voutev, R., Mann, R. S. 2012. A dynamic network of morphogens and transcription factors patterns the fly leg. Curr. Top. Dev. Biol. 98: 173-98.

Griffin, K. J., Stoller, J., Gibson, M., Chen, S., Yelon, D., Stainier, D. Y., Kimelman, D. 2000. A conserved role for H15-related T-box transcription factors in zebrafish and Drosophila heart formation. Dev. Biol. 218: 235-247.

Grimm, S., Pflugfelder, G. O. 1996. Control of the gene optomotor-blind in Drosophila wing development by decapentaplegic and wingless. Science 271: $1601-1604$.

Grossmann, D., Scholten, J., Prpic, N. M. 2009. Separable functions of wingless in distal and ventral patterning of the Tribolium leg. Dev. Genes Evol. 219: 469479.

Held, L. I., Heup, M. A., Sappington, J. M., Peters, S. D. 1994. Interactions of decapentaplegic, wingless, and Distal-less in the Drosophila leg. Roux Arch. Dev. Biol. 203: 310-319.

Henikoff, S., Henikoff, J. G. 1992. Amino acid substitution matrices from protein blocks. Proc. Natl. Acad. Sci. USA 89: 10915-10919.

Inoue, Y., Mito, T., Miyawaki, K., Matsushima, K., Shinmyo, Y., Heanue, T. A., Mardon, G., Ohuchi, H., Noji, S. 2002. Correlation of expression patterns of 
homothorax, dachshund, and Distal-less with the proximodistal segmentation of the cricket leg bud. Mech. Dev. 113: 141-148.

Janssen, R., Prpic, N. M., Damen, W. G. M. 2004. Gene expression suggests decoupled dorsal and ventral segmentation in the millipede Glomeris marginata (Myriapoda: Diplopoda). Dev. Biol. 268: 89-104.

Janssen, R., Damen, W. G. 2008. Diverged and conserved aspects of heart formation in a spider. Evol. Dev. 10: 155-165.

Janssen, R., Feitosa, N. M., Damen, W. G., Prpic, N. M. 2008a. The T-box genes H15 and optomotor-blind in the spiders Cupiennius salei, Tegenaria atrica and Achaearanea tepidariorum and the dorsoventral axis of arthropod appendages. Evol. Dev. 10: 143-154.

Janssen, R., Budd, G. E., Damen, W. G. M., Prpic, N. M. 2008b. Evidence for Wgindependent tergite boundary formation in the millipede Glomeris marginata. Dev. Genes Evol. 218: 361-370.

Janssen, R., Eriksson, B. J., Budd, G. E., Akam, M., Prpic, N. M. 2010. Gene expression patterns in an onychophoran reveal that regionalization predates limb segmentation in pan-arthropods. Evol. Dev. 12: 363-372.

Janssen, R., Budd, G. E. 2013. Deciphering the onychophoran 'segmentation gene cascade': gene expression reveals limited involvement of pair rule gene orthologs in segmentation, but a highly conserved segment polarity gene network. Dev. Biol. 382: 224-234.

Jiang, J., Struhl, G. 1996. Complementary and mutually exclusive activities of Decapentaplegic and Wingless organize axial patterning during Drosophila leg development. Cell 86: 401-409.

Jockusch, E. L., Nulsen, C., Newfeld, S. J., Nagy, L. M. 2000. Leg development in 
flies versus grasshoppers: differences in $d p p$ expression do not lead to differences in the expression of downstream components of the leg patterning pathway. Development 127: 1617-1626.

Lecuit, T., Cohen, S. M. 1997. Proximal-distal axis formation in the Drosophila leg. Nature 388: 139-145.

Maves, L., Schubiger, G. 1998. A molecular basis for transdetermination in Drosophila imaginal discs: interactions between wingless and decapentaplegic signaling. Development 125:115-124.

Mayer, G. 2006. Origin and differentiation of nephridia in the Onychophora provide no support for the Articulata. Zoomorphology $125: 1-12$

Mayer, G., Whitington, P. M. 2009. Neural development in Onychophora (velvet worms) suggests a step-wise evolution of segmentation in the nervous system of Panarthropoda. Dev. Biol. 335: 263-275.

Miskolczi-McCallum, C. M., Scavetta, R. J., Svendsen, P. C., Soanes, K. H., Brook, W. J. 2005. The Drosophila melanogaster T-box genes midline and H15 are conserved regulators of heart development. Dev. Biol. 278: 459-472.

Miyawaki, K., Mito, T., Sarashina, I., Zhang, H., Shinmyo, Y., Ohuchi, H., Noji, S. 2004 Involvement of Wingless/Armadillo signaling in the posterior sequential segmentation in the cricket, Gryllus bimaculatus (Orthoptera), as revealed by RNAi analysis. Mech. Dev. 121: 119-130.

Morata, G., Lawrence, P. A. 1975. Control of compartment development by the engrailed gene in Drosophila. Nature 255: 614-617.

Morimura, S., Maves, L., Chen, Y., Hoffmann, F. M. 1996. decapentaplegic overexpression affects Drosophila wing and leg imaginal disc development and wingless expression. Dev. Biol. 177: 136-151. 
Nellen, D., Burke, R., Struhl, G., Basler, K. 1996. Direct and long-range action of a DPP morphogen gradient. Cell 85: 357-368.

Niwa, N., Inoue, Y., Nozawa, A., Saito. M., Misumi, Y., Ohuchi, H., Yoshioka, H., Noji, S. 2000. Correlation of diversity of leg morphology in Gryllus bimaculatus (cricket) with divergence in $d p p$ expression pattern during leg development. Development 127: 4373-4381.

Nulsen, C., Nagy, L. M. 1999. The role of wingless in the development of multibranched crustacean limbs. Dev. Genes Evol. 209: 340-348.

Ober, K. A., Jockusch, E. L. 2006. The roles of wingless and decapentaplegic in axis and appendage development in the red flour beetle, Tribolium castaneum. Dev. Biol. 294: 391-405.

Oliveira Ide, S., Read, V. M., Mayer, G. 2012. A world checklist of Onychophora (velvet worms), with notes on nomenclature and status of names. Zookeys 211: $1-70$.

Pechmann, M., Prpic, N. M. 2009. Appendage patterning in the South American bird spider Acanthoscurria geniculata (Araneae: Mygalomorphae). Dev. Genes Evol. 219: 189-198.

Prpic, N. M., Wigand, B., Damen, W. G., Klingler, M. 2001. Expression of dachshund in wild-type and Distal-less mutant Tribolium corroborates serial homologies in insect appendages. Dev. Genes Evol. 211: 467-477.

Prpic, N. M., Janssen, R., Wigand, B., Klingler, M., Damen, W. G. M. 2003. Gene expression in spider appendages reveals reversal of exd/hth spatial specificity, altered leg gap gene dynamics, and suggests divergent distal morphogen signaling. Dev. Biol. 264: 119-140.

Prpic, N. M., Tautz, D. 2003. The expression of the proximodistal axis patterning 
genes Distal-less and dachshund in the appendages of Glomeris marginata (Myriapoda: Diplopoda) suggests a special role of these genes in patterning the head appendages. Dev. Biol. 260: 97-112.

Prpic, N. M. 2004. Homologs of wingless and decapentaplegic display a complex and dynamic expression profile during appendage development in the millipede Glomeris marginata (Myriapoda: Diplopoda). Front. Zool. 1: 6.

Prpic, N. M., Janssen, R., Damen, W. G., Tautz, D. 2005. Evolution of dorsal-ventral axis formation in arthropod appendages: H15 and optomotor-blind/bifid-type T-box genes in the millipede Glomeris marginata (Myriapoda: Diplopoda). Evol. Dev. 7: 51-57.

Sanchez-Salazar, J., Pletcher, M. T., Bennett, R. L., Brown, S. J., Dandamudi, T. J., Denell, R. E., Doctor, J. S. 1996. The Tribolium decapentaplegic gene is similar in sequence, structure, and expression to the Drosophila dpp gene. Dev. Genes Evol. 206: 237-246.

Schaeper, N. D., Wimmer, E. A., Prpic, N. M. 2013. Appendage patterning in the primitively wingless hexapods Thermobia domestica (Zygentoma: Lepismatidae) and Folsomia candida (Collembola: Isotomidae). Dev. Genes Evol. 223: 341-350.

Sharma, P. P., Schwager, E. E., Extavour, C. G., Giribet, G. 2012. Evolution of the chelicera: a dachshund domain is retained in the deutocerebral appendage of Opiliones (Arthropoda, Chelicerata). Evol. Dev. 14: 522-533.

Strimmer, K., von Haeseler, A. 1996. Quartet puzzling: a quartet maximum likelihood method for reconstructing tree topologies. Mol. Biol. Evol. 13: 964-969.

Struhl, G., Basler, K. 1993. Organizing activity of wingless protein in Drosophila. Cell 72: 527-540. 
Svendsen, P. C., Formaz-Preston, A., Leal, S. M., Brook, W. J. 2009. The Tbx20 homologs midline and H15 specify ventral fate in the Drosophila melanogaster leg. Development 136: 2689-2693.

Swofford, D. L. 2002. PAUP. Phylogenetic Analysis Using Parsimony (*and Other Methods), Version 4. Sinauer Associates, Sunderland, MA.

Tanaka, K., Truman, J. W. 2007. Molecular patterning mechanism underlying metamorphosis of the thoracic leg in Manduca sexta. Dev. Biol. 305: 539-550.

Thompson, J. D., Gibson, T. J., Plewniak, F., Jeanmougin, F., Higgins, D. G. 1997. The CLUSTAL_X windows interface: flexible strategies for multiple sequence alignment aided by quality analysis tools. Nucleic Acids Res. 25: 4876-4882.

Treffkorn, S., Mayer, G. 2013. Expression of the decapentaplegic ortholog in embryos of the onychophoran Euperipatoides rowelli. Gene Expr. Patterns 13: 384394.

Walker, M., Tait, N. N. 2004. Studies of embryonic development and the reproductive cycle in ovoviviparous Australian Onychophora (Peripatopsidae). J. Zool. (Lond.) 264: 333-354.

Wilder, E. L., Perrimon, N. 1995. Dual functions of wingless in the Drosophila leg imaginal disc. Development 121: 477-488.

Yamamoto, D. S., Sumitani, M., Tojo, K., Lee, J. M., Hatakeyama, M. 2004. Cloning of a decapentaplegic orthologue from the sawfly Athalia rosae (Hymenoptera), and its expression in the embryonic appendages. Dev. Genes Evol. 214: 128-133.

Zecca, M., Basler, K., Struhl, G. 1996. Direct and long-range action of a Wingless morphogen gradient. Cell 87: 833-844. 


\section{LEGENDS}

Fig. 1 Expression of Euperipatoides decapentaplegic

Orientation is with anterior to the left in A and B, and anterior up in C-E. A represents one half of a flat-mounted embryo. Lateral view. The asterisk marks the dorsal ectoderm of the frontal appendage that is free from strong $d p p$-expression. The arrow and the arrowhead indicate two domains of enhanced expression in the developing ventral nervous system. B Anterior part of an embryo. Ventral view. Arrows indicate strong expression in the tips of the walking limbs. C Ventral view. Arrows as in C. Note that the anterior ectoderm of the limbs is free from $d p p$-expression (asterisks). D Ventral view on a slime papilla. Asterisk as in D. E Dorsal view on the first walking limb. Asterisk and arrow as in D. Abbreviations: fap, frontal appendage; hl, head lobe; L1, first walking limb; pp, posterior pit; sp, slime papilla; st, stage.

Fig. 2 Expression of Euperipatoides wingless

Anterior is to the left. A Arrowhead indicates lack of expression in the central and proximal part of a walking limb. Arrow indicates continuous expression along the ventral ectoderm at an early stage of limb outgrowth. $\mathbf{A}^{\prime}$ represents DAPI counter staining of the same embryo as in A. Abbreviations as in Fig. 1 and j, jaw.

Fig. 3 Expression of Euperipatoides optomotor-blind1

In all panels, except panel A, anterior is to the left. In panel A three embryos with different orientation are shown. A The two upper embryos represent lateral views; the lower embryo represents a ventral view. Asterisks mark expression in the posterior pit region. Arrows mark expression in the head lobes. B Lateral view. C Ventral view. D 
Ventral view. Arrowhead indicates expression in the optical region dorsal in the head lobes. E Ventral view on a flat-mounted embryo. Arrowhead as in D. F Flat-mounted embryo. Magnification of the L4 to L6 limbs as seen in E. Expression is in the ectoderm (ect). G Lateral view. Arrow indicates a ring of expression near the tip of the frontal appendage. H Dorsal view. I Same embryo as shown in H, but ventral view. Arrow indicates expression inside the head lobes. Asterisk marks unspecific staining of the cuticle in the mouth. $\mathrm{A}^{\prime}, \mathrm{B}^{\prime}$ and $\mathrm{G}^{\prime}$ represent DAPI-counter stained embryos as shown in A, B and G. Abbreviations as in Fig. 1, and ect, ectoderm; mes, mesoderm.

Fig. 4 Expression of Euperipatoides optomotor-blind2

In all panels anterior is to the left. A Lateral view. Arrowhead indicates expression in the dorsal rim of the heal lobe. B Lateral view. Arrowhead indicates continuous expression that is level with the dorsal primordia of the limbs (and tissue in between). C Lateral view. D Same embryo as shown in C; Ventral view. E One half of a latmounted embryo; lateral view. F Lateral view. Arrows indicate single dots of expression dorsal to the limbs. G Anterior part of an embryo. Ventral view. Arrowhead indicates expression in the ventral nervous system. H Same embryo as in G; dorsal view. Arrowhead indicates weak expression in the distal part of a limb. I Same embryo as in $\mathrm{G}$ and $\mathrm{H}$; lateral view. Arrowheads indicate expression in the outgrowing dorsal tissue (lower arrowhead as arrows in F). $\mathrm{A}^{\prime}, \mathrm{B}^{\prime}$ and $\mathrm{F}^{\prime}$ represent DAPI-counter stained embryos as shown in A, B and F. Abbreviations as in Fig. 1; and e, eye; j, jaw.

Fig. 5 Expression of Euperipatoides $H 15$ 
In all panels anterior is to the left. A Lateral view. B Close-up on the slime papilla to L2 segments of the embryo shown in A. Note that expression in the limbs is restricted to the mesoderm. C Lateral view. Arrows indicate expression in the dorsal tube (heart). Note strong expression in L4 and L5. D Lateral view. Flat-mounted embryo. Arrow as in C. Arrowhead indicates expression in the ventral nervous system. Note strong expression in L4 and L5. C' represents DAPI-counter staining of same embryo as shown in C. Abbreviations as in Fig. 1.

Fig. 6 Summary of expression of $d p p, o m b 1, o m b 1$ and $H 15$ in developing limbs of Euperipatoides

All panels represent lateral views. The base of the limbs is to the left. Dorsal is up. AD Expression of $d p p$ in a stage 16 embryo. E-H Expression of $d p p$ in a stage 20 embryo. I-L Expression of omb1. M-P Expression of omb2. Q-T Expression of H15.

Fig. 7 Schematic comparison of $d p p, o m b, w g$ and $H 15$ expression in onychophorans, chelicerates, myriapods and insects with direct limb development (e.g. Tribolium) and indirect limb development (e.g. Drosophila). In the cartoons, distal is up, dorsal is to the left and ventral is to the right (cf. explanatory drawing). For the expression of $d p p$ in an onychophoran and a myriappod light red represents weaker expression compared to the (stronger) expression in the tips (dark red).

\section{Supplementary Data}

Fig. S1 Overview over anti-sense mRNA probes for the detection of Euperipatoides decapentaplegic (dpp). 
The probe that is marked with an asterisk (*) represents the best-working probe (best ratio of signal to background and fastest staining-time). The 'transcriptome probe' detected the same pattern as the ' 3 'RACE probe'. The short 'PCR probe' did not detect any specific signal.

Fig. S2 Phylogenetic analysis of E. kanangrensis Decapentaplegic and T-box genes The conserved T-box sequences have been used for the analyses. The phylogram represents the unrooted majority rule consensus computed from 1000 intermediate trees produced with the Quartet Puzzling Method (Strimmer and von Haeseler 1996). Numbers indicate the reliability values of the branches. 
Figures

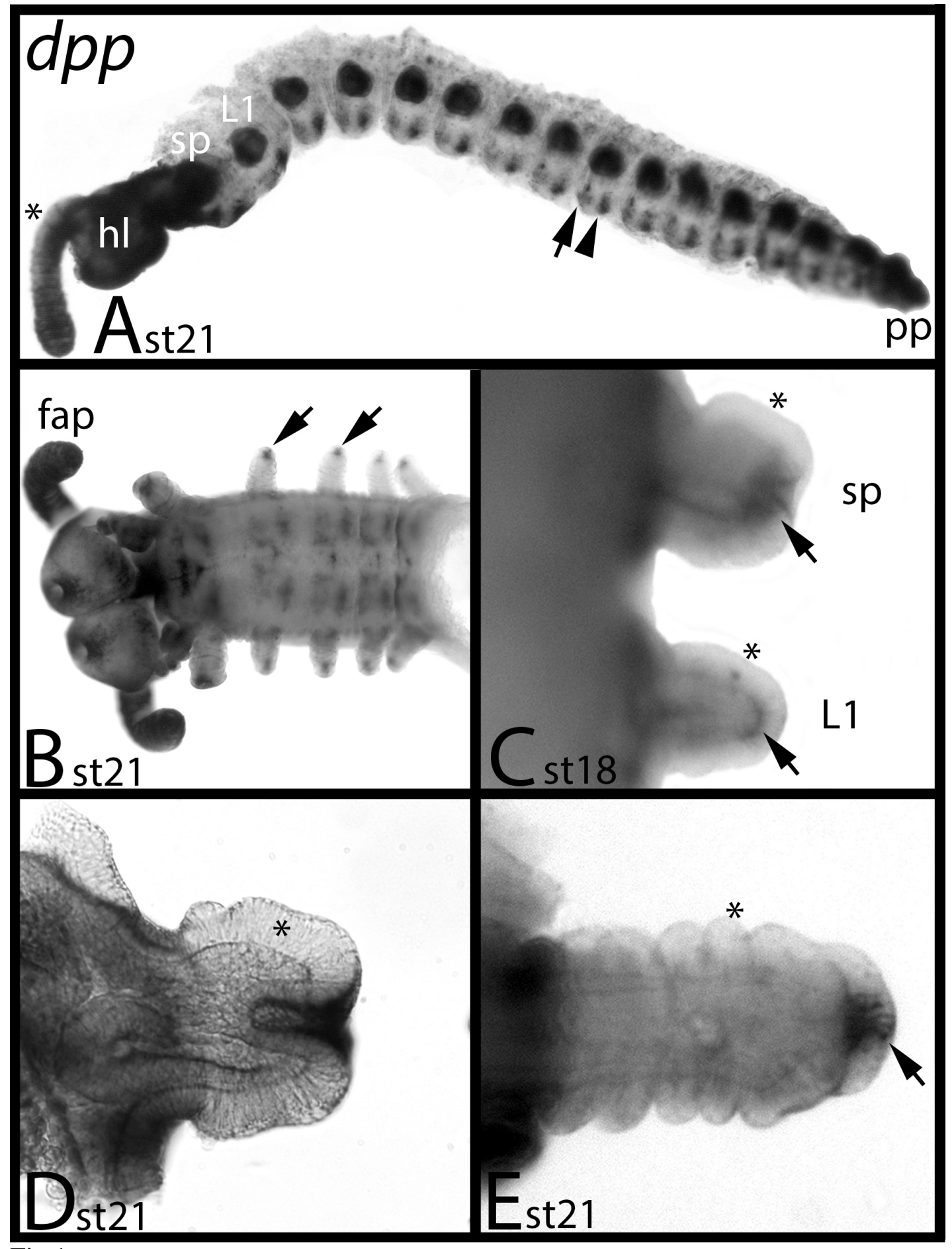

Fig.1 


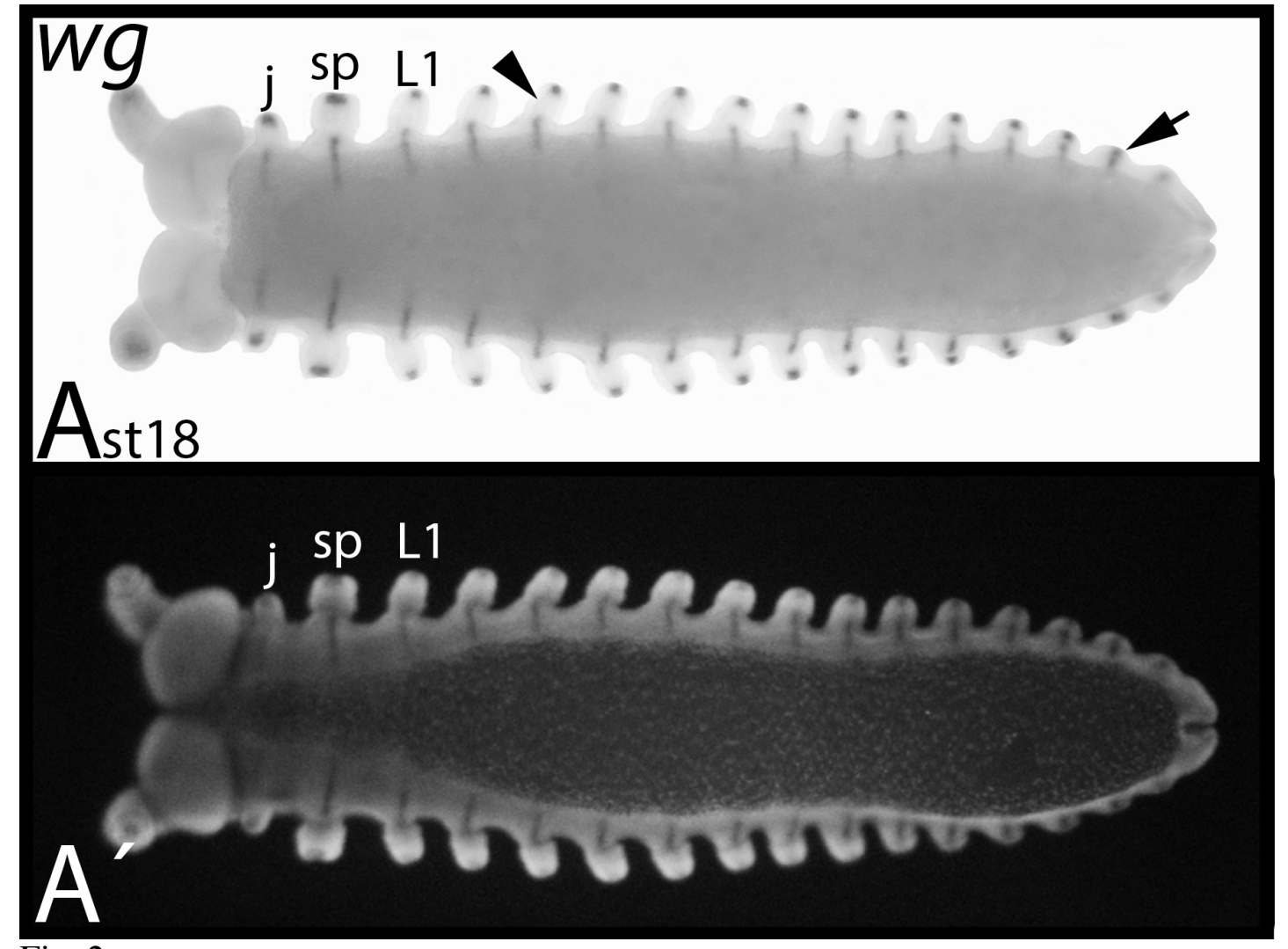

Fig. 2 


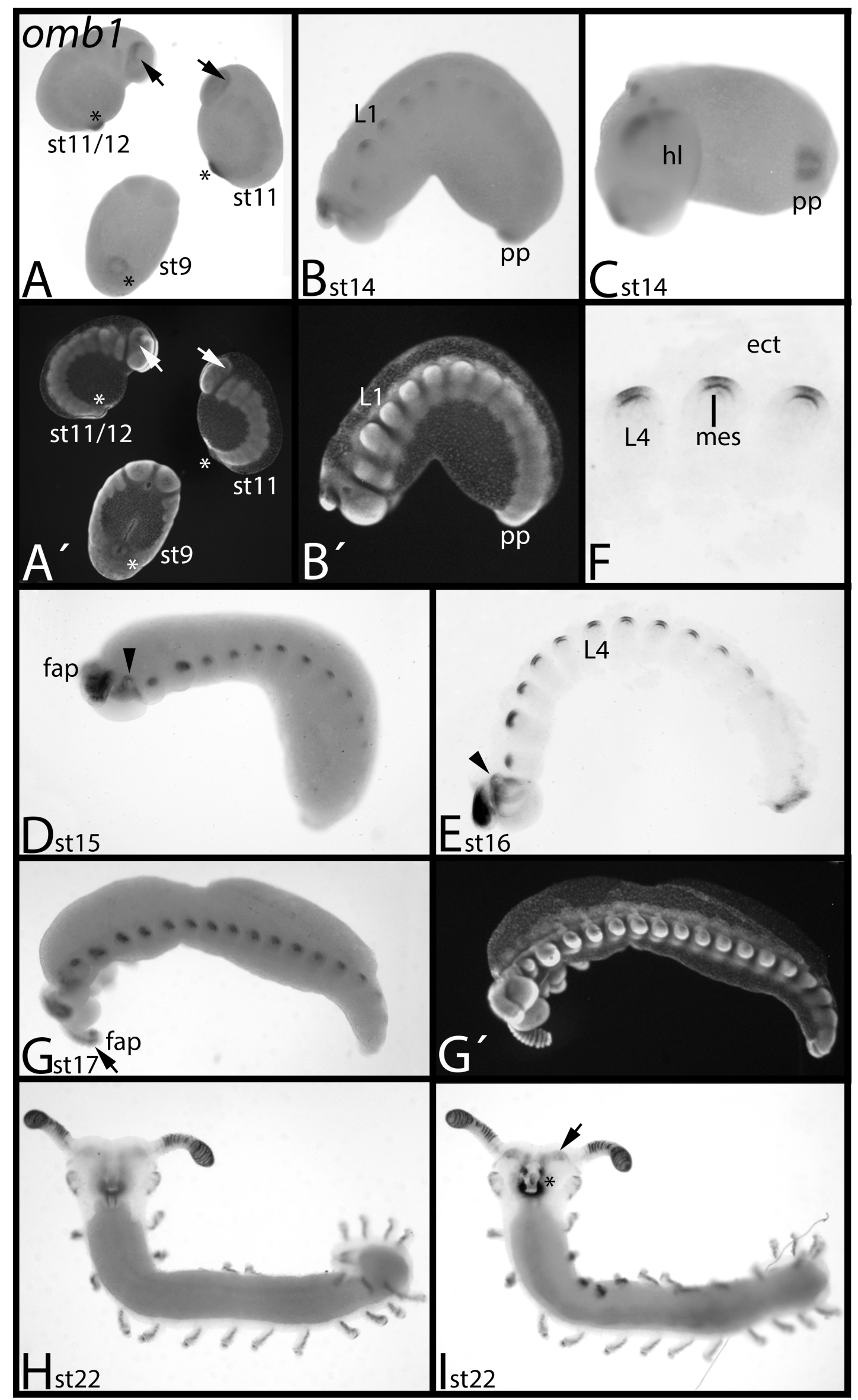

Fig. 3 


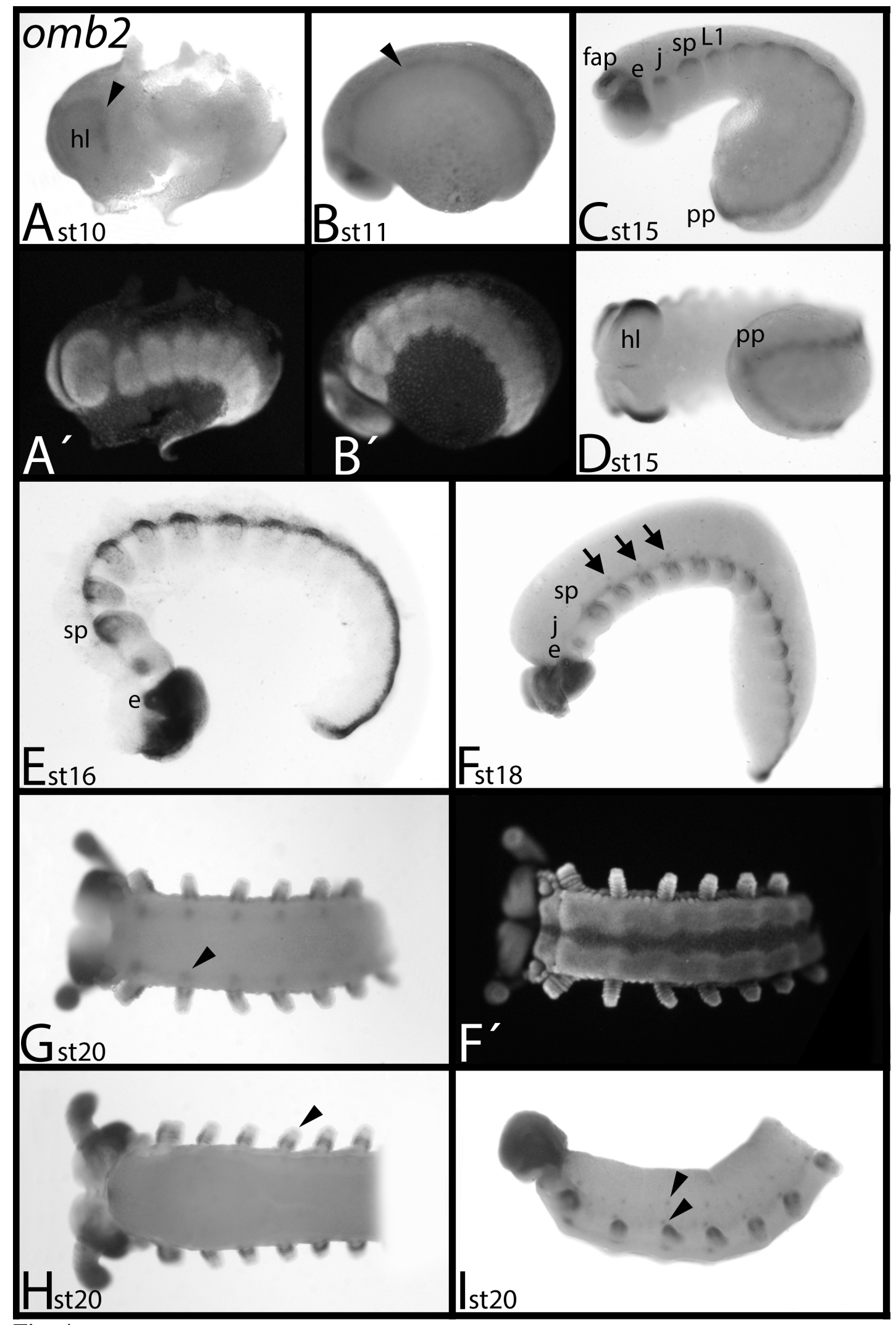

Fig. 4 


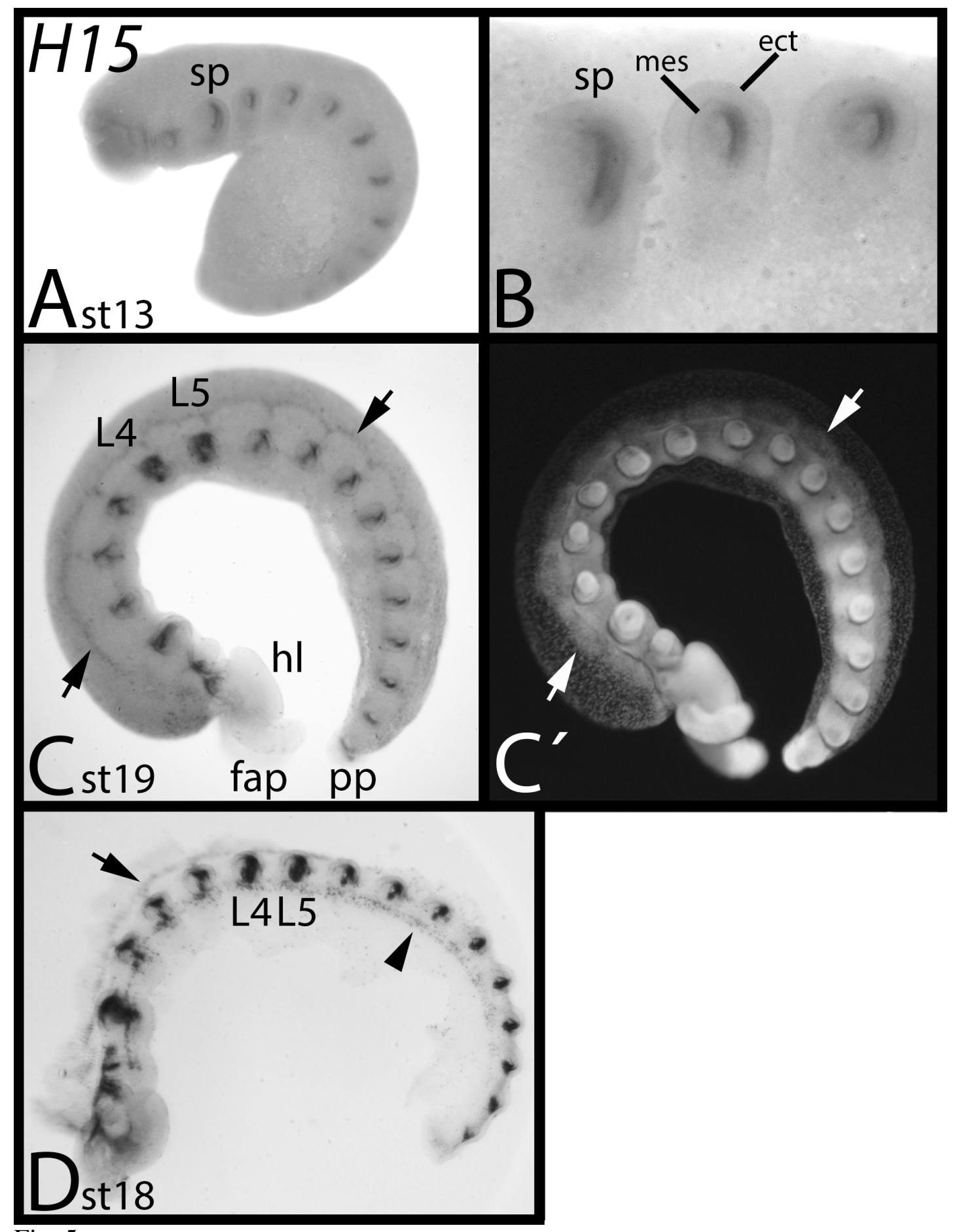

Fig. 5 


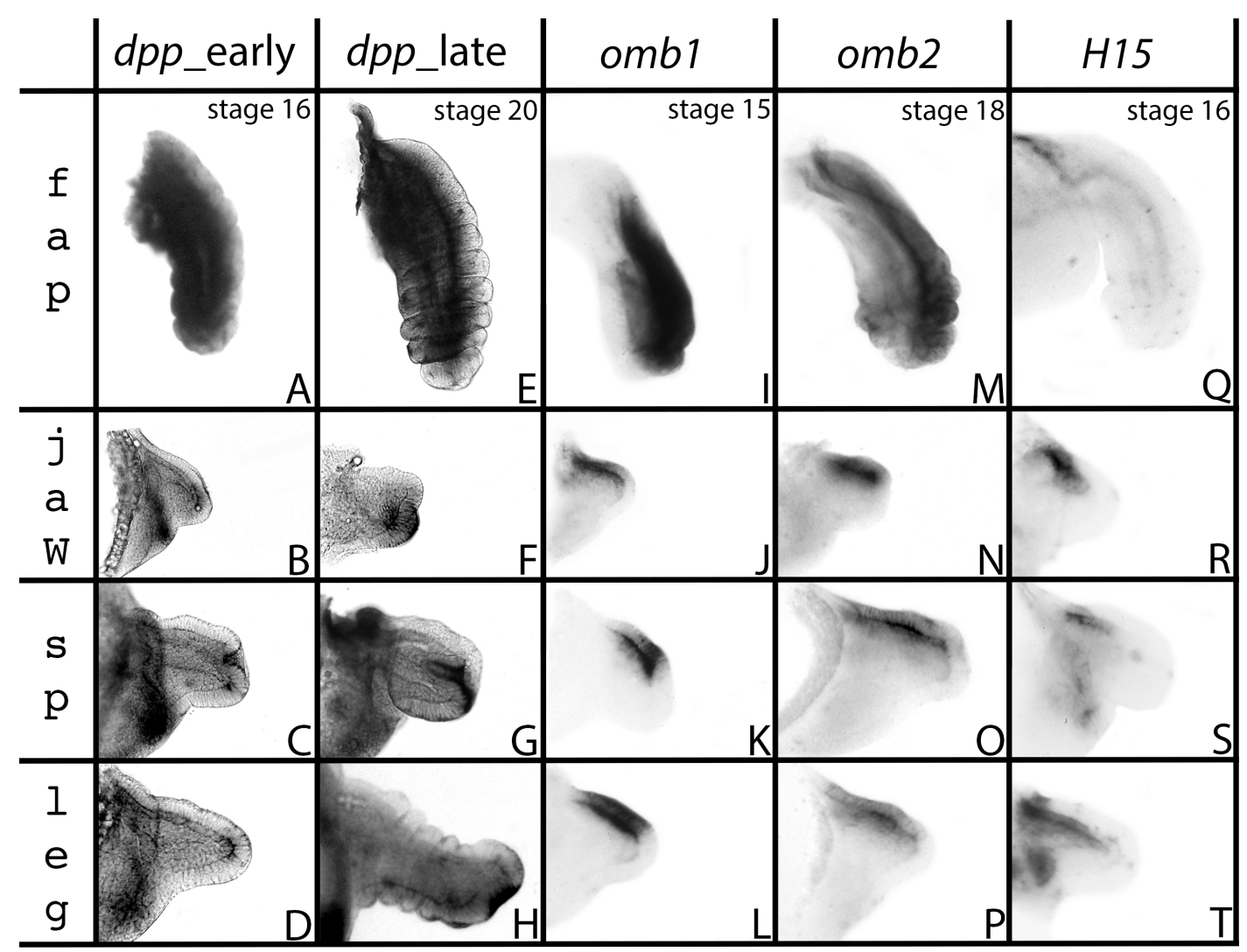

Fig. 6

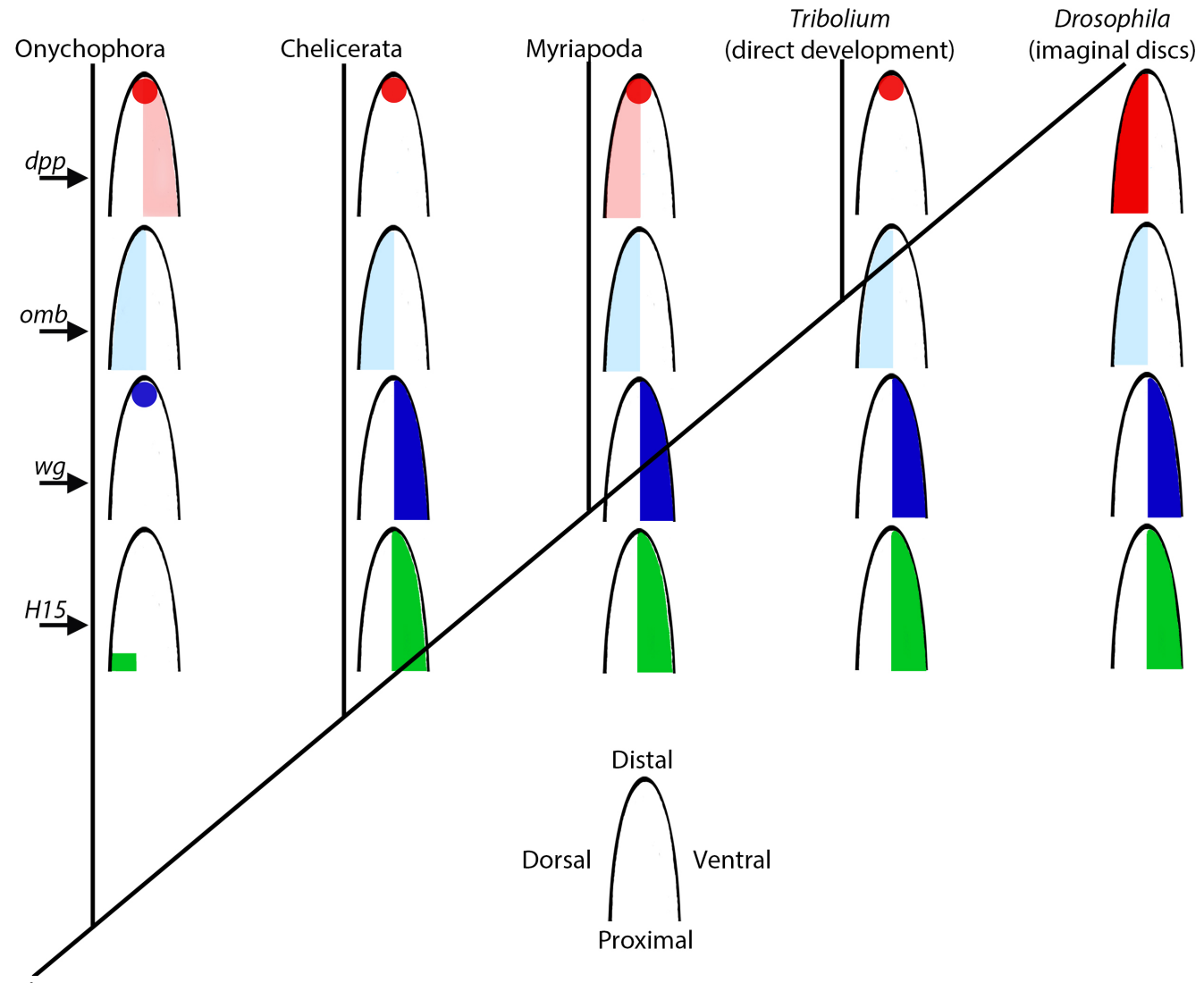

Fig. 7 